\title{
Energy shaping of boundary controlled linear port Hamiltonian systems
}

\author{
Yann Le Gorrec* Alessandro Macchelli** Hector Ramirez* \\ Hans Zwart ${ }^{* * *}$ \\ * Department of Automation and Micro-Mechatronic Systems, \\ FEMTO-ST UMR CNRS 6174, ENSMM, 26 chemin de l'épitaphe, \\ F-25030 Besançon, France. \{ramirez, legorrec\}@emto-st.fr \\ ** Department of Electrical, Electronic and Information Engineering \\ "Guglielmo Marconi" (DEI), University of Bologna, viale del \\ Risorgimento 2, 40136 Bologna, Italy. \\ (e-mail:alessandro.macchelli@unibo.it) \\ *** University of Twente, Faculty of Electrical Engineering, \\ Mathematics and Computer Science, Department of Applied \\ Mathematics, P.O. Box 2177500 AE Enschede, The Netherlands. \\ (e-mail:h.j.zwart@utwente.nl)
}

\begin{abstract}
In this paper, we consider the asymptotic stabilization of a class of one dimensional boundary controlled port Hamiltonian systems by an immersion/reduction approach and the use of Casimir invariants. We first extend existing results on asymptotic stability of linear infinite dimensional systems controlled at their boundary to the case of stable Port Hamiltonian controllers including some physical constraints as clamping. Then the relation between structural invariants, namely Casimir functions, and the controller structure is computed. The Casimirs are employed in the selection of the controllers Hamiltonian to shape the total energy function of the closed loop system and introduce a minimum in the desired equilibrium configuration. The approach is illustrated on the model of a micro manipulation process with full-actuation on one side of the spatial domain.
\end{abstract}

Keywords: Boundary control systems, infinite dimensional port Hamiltonian systems, asymptotic stability, Casimir.

\section{INTRODUCTION}

Boundary controlled distributed parameter systems have been extensively studied in the literature even in the linear case. The derivation of the control law usually goes through an appropriate choice of a Lyapunov function including the boundary variables that are used for control purpose. Lyapunov functions being intrinsically linked to the energy it is quite natural to use a formalism that emphasis the links existing between the energy and the dynamics of the system. In finite dimension this is done by the use of the port Hamiltonian framework and the control by energy shaping (Ortega et al., 2001; van der Schaft, 2004) or IDA-PBC (Ortega et al., 2002). The Port Hamiltonian framework has been extended to the case of infinite dimensional system using a geometric differential point of view in (van der Schaft and Maschke, 2002) and using a functional analysis point of view in the one dimensional case in (Le Gorrec et al., 2005). Such approach allows to link the variation of the energy within the system to the power flow at its boundary. In (Villegas et al., 2005) and (Villegas et al., 2009) this approach has been used to derive some simple matrix conditions to

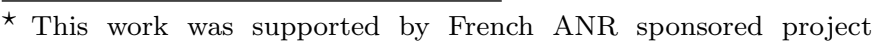
HAMECMOPSYS under Reference Code ANR-11-BS03-0002 and the LABEX ANR-11-LABX-01-01
}

insure the exponential or asymptotic stability for a class of linear 1D boundary controlled systems. Port Hamiltonian formulation has also been used to design stabilizing control laws by energy shaping (Macchelli and Melchiorri, 2004). The idea is to extend the dynamic system state space by the way of the interconexion of a dynamic controller and then to reduce it through the structural invariants, named Casimir invariants, in order to shape the closed loop energy function (that is used as Lyapunov function).

In this paper we consider a class of linear boundary port Hamiltonian systems defined on the one dimensional space interconnected in an energy preserving way to a finite dimensional port Hamiltonian controller and including some physical constraints by the rank deficiency of input/output matrices of the controller. We first prove that the closed loop system is asymptotically stable as soon as the controller is exponentially stable. We then propose some sufficient conditions to derive the closed loop Casimir functions that will be used to link the controller states to the system states. The approach is then applied to the control of a micromanipulation process that is used for the characterization of biological samples. In this case the considered finite dimensional system is composed of the suspension system+biological sample (that are fixed) and of the controller (that we have to design). 


\section{BOUNDARY CONTROLLED PORT-HAMILTONIAN SYSTEMS}

The class of boundary controlled systems we study is described by the following PDE:

$$
\frac{\partial x}{\partial t}=P_{1} \frac{\partial}{\partial z}(\mathcal{L}(z) x(t, z))+\left(P_{0}-G_{0}\right) \mathcal{L}(z) x(t, z),
$$

where $z \in(a, b), P_{1} \in M_{n}(\mathbb{R})\left(M_{n}(\mathbb{R})\right.$ denotes the space of real $n \times n$ matrices) is a non-singular symmetric matrix, $P_{0}=-P_{0}^{\top} \in M_{n}(\mathbb{R}), G_{0} \geq 0 \in M_{n}(\mathbb{R})$ and $x$ takes values in $\mathbb{R}^{n}$. Furthermore, $\mathcal{L}(\cdot) \in L_{2}\left(a, b ; M_{n}(\mathbb{R})\right)$ is a bounded and continuously differentiable matrix-valued function satisfying for all $z \in(a, b), \mathcal{L}(z)=\mathcal{L}(z)^{\top}$ and $\mathcal{L}(z)>m I$, with $m$ independent from $z$. For simplicity $\mathcal{L}(z) x(t, z)$ will be denoted by $(\mathcal{L} x)(t, z)$. The state space is defined as $X=L_{2}\left(a, b ; \mathbb{R}^{n}\right)$ with inner product $\left\langle x_{1}, x_{2}\right\rangle_{\mathcal{L}}=$ $\left\langle x_{1}, \mathcal{L} x_{2}\right\rangle$ and norm $\|x\|_{\mathcal{L}}^{2}=\langle x, x\rangle_{\mathcal{L}}$. Hence $X$ is a Hilbert space. Note that the natural norm on $X$ and the $L_{2}$ norm are equivalent. The reason for selecting this space is that $\|\cdot\|_{\mathcal{L}}^{2}$ is usually related to the energy function of the system. Definition 1. (Le Gorrec et al., 2005) Let $\mathcal{L} x \in H^{1}\left(a, b ; \mathbb{R}^{n}\right)$. The boundary port variables associated with system (1) are the vectors $e_{\partial, \mathcal{L} x}, f_{\partial, \mathcal{L} x} \in \mathbb{R}^{n}$, defined by

$$
\left[\begin{array}{l}
f_{\partial, \mathcal{L} x} \\
e_{\partial, \mathcal{L} x}
\end{array}\right]=\frac{1}{\sqrt{2}}\left[\begin{array}{cc}
P_{1} & -P_{1} \\
I & I
\end{array}\right]\left[\begin{array}{l}
(\mathcal{L} x)(b) \\
(\mathcal{L} x)(a)
\end{array}\right]=R\left[\begin{array}{l}
(\mathcal{L} x)(b) \\
(\mathcal{L} x)(a)
\end{array}\right]
$$

Note that the port variables are linear combinations of the boundary variables.

Theorem 2. (Le Gorrec et al., 2005) Let $W$ be a $n \times 2 n$ real matrix. If $W$ has full rank and satisfies $W \Sigma W^{\top} \geq 0$, where $\Sigma=\left[\begin{array}{ll}0 & I \\ I & 0\end{array}\right]$, then the system (1), with input

$$
u(t)=\mathcal{B} x=W\left[\begin{array}{l}
f_{\partial, \mathcal{L} x}(t) \\
e_{\partial, \mathcal{L} x}(t)
\end{array}\right]
$$

is a boundary control system on $X$. Furthermore, the operator $\mathcal{A} x=P_{1}(\partial / \partial z)(\mathcal{L} x)+\left(P_{0}-G_{0}\right) \mathcal{L} x$ with domain

$$
D(\mathcal{A})=\left\{\mathcal{L} x \in H^{1}\left(a, b ; \mathbb{R}^{n}\right) \mid\left[\begin{array}{l}
f_{\partial, \mathcal{L} x} \\
e_{\partial, \mathcal{L} x}
\end{array}\right] \in \operatorname{ker} W\right\}
$$

generates a contraction semigroup on $X$. Let $\tilde{W}$ be a full rank matrix of size $n \times 2 n$ with $\left[\begin{array}{c}W \\ \tilde{W}\end{array}\right]$ invertible and let $P_{W, \tilde{W}}$ be given by

$$
P_{W, \tilde{W}}=\left(\left[\begin{array}{c}
W \\
\tilde{W}
\end{array}\right] \Sigma\left[\begin{array}{l}
W \\
\tilde{W}
\end{array}\right]^{\top}\right)^{-1}=\left[\begin{array}{ll}
W \Sigma W^{\top} & W \Sigma \tilde{W}^{\top} \\
\tilde{W} \Sigma W^{\top} & \tilde{W} \Sigma \tilde{W}^{\top}
\end{array}\right]^{-1} .
$$

Define the output of the system as the linear mapping $\mathcal{C}: \mathcal{L}^{-1} H^{1}\left(a, b ; \mathbb{R}^{n}\right) \rightarrow \mathbb{R}^{n}$,

$$
y=\mathcal{C} x(t):=\tilde{W}\left[\begin{array}{l}
f_{\partial, \mathcal{L}_{x}}(t) \\
e_{\partial, \mathcal{L}_{x}}(t)
\end{array}\right]
$$

Then for $u \in C^{2}\left(0, \infty ; \mathbb{R}^{k}\right), \mathcal{L} x(0) \in H^{1}\left(a, b ; \mathbb{R}^{n}\right)$, and $u(0)=W\left[\begin{array}{l}f_{\partial, \mathcal{L} x}(0) \\ e_{\partial, \mathcal{L} x}(0)\end{array}\right]$ the following balance equation is satisfied:

$$
\begin{aligned}
\frac{1}{2} \frac{d}{d t}\|x(t)\|_{\mathcal{L}}^{2} & =\frac{1}{2}\left[\begin{array}{l}
u(t) \\
y(t)
\end{array}\right]^{\top} P_{W, \tilde{W}}\left[\begin{array}{l}
u(t) \\
y(t)
\end{array}\right]-\left\langle G_{0} \mathcal{L} x(t), \mathcal{L} x(t)\right\rangle \\
& \leq\left[\begin{array}{l}
(\mathcal{L} x)(t, b) \\
(\mathcal{L} x)(t, a)
\end{array}\right]^{\top}\left[\begin{array}{cc}
P_{1} & 0 \\
0 & -P_{1}
\end{array}\right]\left[\begin{array}{l}
(\mathcal{L} x)(t, b) \\
(\mathcal{L} x)(t, a)
\end{array}\right] .
\end{aligned}
$$

The matrix $P_{W, \tilde{W}}$ is defined only when $\left[\begin{array}{c}W \\ \tilde{W}\end{array}\right]$ is invertible. Notice that in the absence of some internal dissipation
$\left(G_{0}=0\right)$ the system only exchanges energy with the environment through the boundaries since the input and output act on the boundary of the spatial domain.

Remark 3. As it has been pointed out in (Villegas, 2007), if the matrices $W$ and $\tilde{W}$ are selected such that $P_{W, \tilde{W}}=$ $\left[\begin{array}{ll}0 & I \\ I & 0\end{array}\right]=\Sigma$, then the BCS fulfils $\frac{1}{2} \frac{d}{d t}\|x(t)\|_{\mathcal{L}}^{2} \leq u^{\top}(t) y(t)$.

\section{DYNAMIC BOUNDARY CONTROL}

In what follows we consider the feedback loop of Figure 1 where the infinite dimensional system is an impedance passive system as described in Theorem 2 .

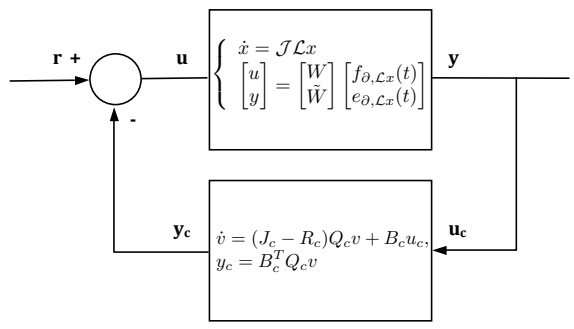

Fig. 1. Power preserving interconnection

This interconnection is power preserving and satisfies:

$$
u=r-y_{c}, \quad u_{c}=y
$$

Furthermore the controller satisfies Assumption 4.

Assumption 4. We consider a controllable, observable and passive port Hamiltonian controller on the form:

$$
\left\{\begin{array}{l}
\dot{v}=\left(J_{c}-R_{c}\right) Q_{c} v+B_{c} u_{c} \\
y_{c}=B_{c}^{T} Q_{c} v
\end{array}\right.
$$

with state space $v \in V=\mathbb{R}^{m}$, input values $u_{c} \in U_{c}=\mathbb{R}^{n}$ and output values $y_{c} \in Y=\mathbb{R}^{n}$. Moreover $J_{c}, R_{c}, Q_{c}$ and $B_{c}$ are constant real matrices of dimension $m \times m$, $m \times m, m \times m$, and $m \times n$, respectively with $J_{c}=-J_{c}^{T}$, $R_{c}=R_{c}^{T} \geq 0$ and $Q_{c}>0$ such that $\left(J_{c}-R_{c}\right) Q_{c}$ is Hurwitz. Proposition 1. From Kalman-Yakubovich-Popov Lemma (Willems, 1972) there exists a symmetric matrix $Q_{d} \in \mathbb{R}^{m}$, $Q_{d}=Q_{d}^{T}>0$ such that:

$$
Q_{c}\left(J_{c}-R_{c}\right) Q_{c}+Q_{c}\left(J_{c}-R_{c}\right)^{T} Q_{c}=-Q_{d}
$$

\section{ASYMPTOTIC STABILITY}

To prove the asymptotic stability of the closed loop system of Figure 1 we first prove the closed loop operator generates a contraction semigroup on an extended space. Then we prove that from contraction properties the solutions converge to an invariant set. Finally we show this invariant set reduces to a unique point, proving the asymptotic stability of the closed loop system.

Theorem 5. (Villegas et al., 2005) Let the state of the open-loop BCS satisfy $\frac{1}{2} \frac{d}{d t}\|x(t)\|_{\mathcal{L}}^{2} \leq u^{\top}(t) y(t)$. Consider a LTI finite dimensional system with storage function $E_{c}(t)=\frac{1}{2}\left\langle v(t), Q_{c} v(t)\right\rangle_{\mathbb{R}^{m}}, Q_{c}=Q_{c}^{\top} \geq 0 \in \mathbb{R}^{m} \times \mathbb{R}^{m}$ satisfying Assuption 4. Then the feedback interconnection of the BCS and the finite dimensional system is again a BCS on the extended state space $\tilde{x} \in \tilde{X}=X \times V$ 
with inner product $\left\langle\tilde{x}_{1}, \tilde{x}_{2}\right\rangle_{\tilde{X}}=\left\langle x_{1}, x_{2}\right\rangle_{\mathcal{L}}+\left\langle v_{1}, Q_{c} v_{2}\right\rangle_{V}$. Furthermore, the operator $\mathcal{A}_{e}$ defined by

$$
\mathcal{A}_{e} \tilde{x}=\left[\begin{array}{cc}
\mathcal{J} \mathcal{L} & 0 \\
B_{c} \mathcal{C} & \left(J_{c}-R_{c}\right) Q_{c}
\end{array}\right]\left[\begin{array}{l}
x \\
v
\end{array}\right]
$$

with

$$
D\left(\mathcal{A}_{e}\right)=\left\{\left[\begin{array}{l}
x \\
v
\end{array}\right] \in\left[\begin{array}{l}
X \\
V
\end{array}\right] \mid \mathcal{L} x \in H^{N}\left(a, b ; \mathbb{R}^{n}\right),\left[\begin{array}{c}
f_{\partial, \mathcal{L} x} \\
e_{\partial, \mathcal{L} x} \\
v
\end{array}\right] \in \operatorname{ker} \tilde{W}_{D}\right\},
$$

where

$$
\tilde{W}_{D}=\left[\begin{array}{ll}
W & B_{c}^{T} Q_{c}
\end{array}\right]
$$

generates a contraction semigroup on $\tilde{X}$.

Proof. The proof is similar to the one presented in (Villegas, 2007, Theorem 5.8, pp:120) but in the case of the use of a Port Hamiltonian structure for the controller. We also consider that a subset of the boundary conditions of the infinite dimensional system can be set to zero through a rank deficiency of $B_{c}$. The proof is performed in two steps. First we have to prove that there exists an operator $\mathscr{B} \in \mathcal{L}(U, \tilde{X})$ such that for all $u \in U, \mathscr{B} u \in D\left(\mathcal{A}_{e}\right) \times$ $\mathbb{R}^{n}$, and $\left[\mathcal{B} \mathcal{C}_{c}\right] \mathscr{B} u=u$. Such operator exists as soon as $\tilde{W}_{D}$ is full rank. In the present case the condition is satisfied as $W$ is full rank. Secondly we need to prove that $\mathcal{A}_{e}$ generates a semigroup. For that we use the LümerPillips theorem (Jacob and Zwart, 2012, Theorem 6.1.7, pp:69) which is divided in two parts: showing that $\mathcal{A}_{e}$ is a dissipative operator (i.e. $\operatorname{Re}\left\langle\mathcal{A}_{e} \tilde{x}, \tilde{x}\right\rangle \leq 0$ ) and that $\operatorname{ran}\left(I-\mathcal{A}_{e}\right)=\tilde{X}=X \times V$. Let consider $\omega=\left[\begin{array}{l}x \\ v\end{array}\right] \in D\left(\mathcal{A}_{e}\right)$ then we have $(\tilde{X}$ is a real Hilbert space equiped with the product $\left.\left\langle\tilde{x}_{1}, \tilde{x}_{2}\right\rangle_{\tilde{X}}=\left\langle x_{1}, x_{2}\right\rangle_{\mathcal{L}}+\left\langle v_{1}, Q_{c} v_{2}\right\rangle_{V}\right)$ :

$$
\left\langle\mathcal{A}_{e} \omega, \omega\right\rangle_{\tilde{X}}=\langle\mathcal{J} \mathcal{L} x, x\rangle_{\mathcal{L}}+\left\langle\left(J_{c}-R_{c}\right) v+B_{C}, Q_{c} v\right\rangle_{V}
$$

After some computation and using Equations (3), (4), and (8) the product can be written:

$$
\left\langle\mathcal{A}_{e} \omega, \omega\right\rangle_{\tilde{X}}=-v^{T} Q_{d} v \leq 0
$$

The second part of the proof, $\operatorname{ran}\left(I-\mathcal{A}_{e}\right)=\tilde{X}$, follows as soon as the matrix $\left(I-\left(J_{c}-R_{c}\right) Q_{c}\right)$ is non-singular. This is true as all the eigenvalues of the matrix $\left(J_{c}-R_{c}\right) Q_{c}$ are in the left half of the complex plane.

The closed loop system can be written:

$$
\begin{array}{r}
\dot{\tilde{x}}=\mathcal{A}_{e} \tilde{x}, \tilde{x}(0) \in \tilde{X} \\
r(t)=\left[\begin{array}{ll}
\mathcal{B} & B_{c}^{T} Q_{c}
\end{array}\right] \tilde{x} \\
y(t)=\left[\begin{array}{ll}
\mathcal{C} & 0
\end{array}\right] \tilde{x}
\end{array}
$$

Theorem 6. Consider the controller satisfying Assumption 4 connected to the impedance passive system as in Figure 1. Then the operator $\mathcal{A}_{e}$ described in Theorem 5 has compact resolvant.

\section{Proof. See (Villegas, 2007, Theorem 5.9, pp:122)}

It is then possible to prove the asymptotic stability in case of exponentially stable controller of the form (7).

Theorem 7. Consider the feedback system of Figure 1 where the controller is chosen satisfying Assumption 4. Then the closed loop system (1) such that $r=0$ is globally asymptotically stable.

Proof. Let first consider that $\omega(0) \in D\left(\mathcal{A}_{e}\right)$. By Theorem 5 , we know that $\mathcal{A}_{e}$ generates a contraction semigroup. Let now consider the energy as Lyapunov function $E_{c}(t)=$ $\frac{1}{2}\langle\omega(t), \omega(t)\rangle_{\tilde{X}}$. Since $\omega(0) \in D\left(\mathcal{A}_{e}\right)$ we know that $\omega(t)$ is differentiable and we can derive after some simple computation:

$$
\frac{d E_{c}(t)}{d t}=\langle\dot{\omega}(t), \omega(t)\rangle_{\tilde{X}}=\left\langle\mathcal{A}_{e} \omega(t), \omega(t)\right\rangle_{\tilde{X}}=-v^{T} Q_{d} v
$$

where $Q_{p}>0$. Since $\left(\lambda I-\mathcal{A}_{e}\right)^{-1}$ is compact and the semigroup is a contraction it follows from LaSalle's invariance principle that all solutions of 12 asymptotically tend to the maximal invariant set $\mathcal{O}_{c}=\left\{\tilde{x} \in \tilde{X} \mid \dot{E}_{c}=0\right\}$. Let $\mathcal{E}$ be the largest invariant subset of $\mathcal{O}_{c}$. We are now going to prove that $\mathcal{E}=\{0\}$. From $\dot{E}_{c}(t)=0$ and (13) we have $v(t)=0$ and then $\dot{v}(t)=0$. Let $\eta<n$ be the rank of $\operatorname{ker}\left(B_{c}\right)$. Form $(7) y_{c}=0$ and $n-\eta>0$ components of $u_{c}$ equal 0 . It follows that $\mathcal{O}_{c}$ reduces to the solution of a first order PDE of dimension $n$ with $2 n-\eta$ boundary variables set to zero. It follows from Holmgren's Theorem that $\tilde{x}(t)=0$, hence the asymptotic stability. The same hold for $\omega(0) \in \tilde{X}$ by using denseness argument (John, 1978).

\section{ENERGY SHAPING}

In the case of power preserving interconnection at the boundary of the form (6), the closed loop Hamiltonian function is equal to the sum of the Hamiltonians of the open-loop system (plant) and the controller (Macchelli et al., 2009; Macchelli and Melchiorri, 2004; Macchelli, 2012): $\tilde{E}(x, v)=E(x)+E_{c}(v)$. In order to use this closed loop Hamiltonian as Lyapunov function, one has to guarantee that its minimum is at the desired equilibrium $\frac{\partial \tilde{E}}{\partial x}\left(x^{*}\right)=0$. For this purpose, and in a similar manner as for control of finite dimensional port-Hamiltonian systems (van der Schaft, 2000), it is possible to relate the state variables of the controller with the state variables of the plant by using structural invariants (i.e., which do not depend on the Hamiltonian) named Casimir functions. Indeed, if it is possible to find Casimirs of the form $C(x, v)=v-F(x)$, with $F(x)$ some smooth well defined function of $x$, then on every invariant manifold defined by $v-F(x)=\kappa$, with $\kappa \in \mathbb{R}$ a constant which depends on the initial states of plant and controller, the closedloop Hamiltonian may be written as $\tilde{E}(x, v)=E(x)+$ $E_{c}(F(x)+\kappa)$. The closed-loop Hamiltonian may then be shaped by an appropiate choice of $E_{c}$.

In the following we give sufficient conditions such that Casimir functions exist in the case of closed loop control with dissipative port Hamiltonian controller.

Definition 8. (Macchelli and Melchiorri, 2004; Macchelli, 2012) Consider the BCS defined by Theorem 2 with $r=0$. A function $C: X \times V \rightarrow \mathbb{R}$ is a Casimir function if $\dot{C}=0$ along the solutions for every possible choice of $\mathcal{L}(\cdot)$ and $Q_{c}$.

Following (Macchelli, 2012) we will look for linear Casimir functions in the form

$$
C(x(t), v(t))=\Gamma^{\top} v(t)+\int_{a}^{b} \Psi^{\top}(z) x(t, z) d z
$$

with $\Gamma \in \mathbb{R}^{m}, \Psi(z) \in \mathbb{R}^{n}$ and $\Psi^{\top}(z) x(t, z) \in H^{1}\left(a, b ; \mathbb{R}^{n}\right)$. 
Proposition 2. Consider the BCS defined by Theorem 2 with $r=0$, and with (7) as controller. Then (14) is a Casimir function for the extended system defined by Theorem 5 if:

$$
\begin{array}{r}
P_{1} \frac{\partial}{\partial z} \Psi(z)+\left(P_{0}+G_{0}\right) \Psi(z)=0 \\
\left(J_{c}+R_{c}\right) \Gamma+B_{c} \tilde{W} R\left[\begin{array}{l}
\Psi(b) \\
\Psi(a)
\end{array}\right]=0 \\
B_{c}^{\top} \Gamma+W R\left[\begin{array}{l}
\Psi(b) \\
\Psi(a)
\end{array}\right]=0 .
\end{array}
$$

Proof. The time derivative of the Casimir function is given by

$$
\begin{aligned}
\frac{d}{d t} C= & \Gamma^{\top}\left[\left(J_{c}-R_{c}\right) Q_{c} v+B_{c} u_{c}\right] \\
& +\int_{a}^{b} \Psi^{\top}\left[P_{1} \frac{\partial}{\partial z}(\mathcal{L} x)+\left(P_{0}-G_{0}\right)(\mathcal{L} x)\right] d z
\end{aligned}
$$

The Casimir function has to be independent from $\mathcal{L}(\cdot)$ and $Q_{c}$, and on other hand the power preserving interconnection introduces some constraint on the possible energy functions. To this end it is convenient to "parametrize" the boundary port variables $\left(f_{\partial, \mathcal{L} x}, e_{\partial, \mathcal{L} x}\right)$. Since the matrix $\left[\begin{array}{ll}W & \tilde{W}\end{array}\right]$ is invertible and $P_{W, \tilde{W}}=\Sigma$, we may define $\left[f_{\partial, \mathcal{L} x}^{\top}, e_{\partial, \mathcal{L} x}^{\top}\right]^{\top}=\Sigma\left(W^{\top} \gamma_{1}+\tilde{W}^{\top} \gamma_{2}\right)$, with $\gamma_{1}, \gamma_{2} \in \mathbb{R}^{n}$. Recalling the definition of $u$ and $y$ (Theorem 2) and $u^{\top} y+$ $u_{c}^{\top} y_{c}=0$ we have $u_{c}=\gamma_{1}$ and $B_{c}^{\top} Q_{c} v=-\gamma_{2}$, which implies

$$
\left[\begin{array}{l}
f_{\partial, \mathcal{L} x} \\
e_{\partial, \mathcal{L} x}
\end{array}\right]=\Sigma W^{\top} \gamma_{1}-\Sigma \tilde{W}^{\top} B_{c}^{\top} Q_{c} v
$$

Hence, the boundary port variables, and by Definition 1 also $(\mathcal{L} x)(a)$ and $(\mathcal{L} x)(b)$, are characterized by $\gamma_{1}$ and $Q_{c}$. The integral term in (18) may be written as

$$
\begin{aligned}
& \Psi^{\top}\left[P_{1} \frac{\partial}{\partial z}(\mathcal{L} x)+\left(P_{0}-G_{0}\right)(\mathcal{L} x)\right] \\
& =\frac{\partial}{\partial z}\left[(\mathcal{L} x)^{\top} P_{1} \Psi\right]-(\mathcal{L} x)^{\top}\left[P_{1} \frac{\partial}{\partial z} \Psi+\left(P_{0}+G_{0}\right) \Psi\right] .
\end{aligned}
$$

Using (19) and $u_{c}=\gamma_{1}$, we may write (18) as

$$
\begin{aligned}
\frac{d}{d t} C=-v^{\top} Q_{c}\left(J_{c}+R_{c}\right) \Gamma+\gamma_{1}^{\top} B_{c}^{\top} \Gamma & \\
-\int_{a}^{b}(\mathcal{L} x)^{\top} & {\left[P_{1} \frac{\partial}{\partial z} \Psi+\left(P_{0}+G_{0}\right) \Psi\right] d z } \\
+ & {\left[\begin{array}{c}
(\mathcal{L} x)(b) \\
(\mathcal{L} x)(a)
\end{array}\right]^{\top} R^{\top} \Sigma R\left[\begin{array}{l}
(\mathcal{L} x)(b) \\
(\mathcal{L} x)(a)
\end{array}\right] }
\end{aligned}
$$

where $R^{\top} \Sigma R=\left[\begin{array}{cc}P_{1} & 0 \\ 0 & -P_{1}\end{array}\right]$. The integral term vanishes for any $\mathcal{L}$ if and only if $\Psi$ satisfies (15). Furthermore using (2), (19) and $\Sigma \Sigma=I$ we have

$$
\begin{aligned}
{\left[\begin{array}{l}
(\mathcal{L} x)(b) \\
(\mathcal{L} x)(a)
\end{array}\right]^{\top} R^{\top} \Sigma R\left[\begin{array}{l}
\Psi(b) \\
\Psi(a)
\end{array}\right]=} & \\
& \left\{\gamma_{1}^{\top} W-v^{\top} Q_{c} B_{c} \tilde{W}\right\} R\left[\begin{array}{l}
\Psi(b) \\
\Psi(a)
\end{array}\right],
\end{aligned}
$$

from which (16) and (17) follows.

Remark 9. Under the hypothesis of the previous proposition, assume that $\hat{\Gamma}=\left[\Gamma_{1}, \ldots, \Gamma_{m}\right]=-I$. In this way one has in closed-loop that $v_{i}(t)=\int_{a}^{b} \Psi_{i}^{\top}(z) x(t, z) d z+\kappa_{i}$, $i=1, \ldots, m$, with $\kappa_{i} \in \mathbb{R}$ a constant that only depends on the initial conditions. Under this hypothesis the Hamiltonian function of controller becomes a function of the state variables of the plant, and may be chosen to obtain a desired stability profile in closed-loop, namely a (possibly) global minimum at the desired equilibrium configuration.

\section{DNA-MANIPULATION PROCESS}

In this section we focus on the control of a nanotweezer used for DNA manipulation (Boudaoud et al., 2012). For this control design a very simple model of the tweezers is presented in Figure 2.

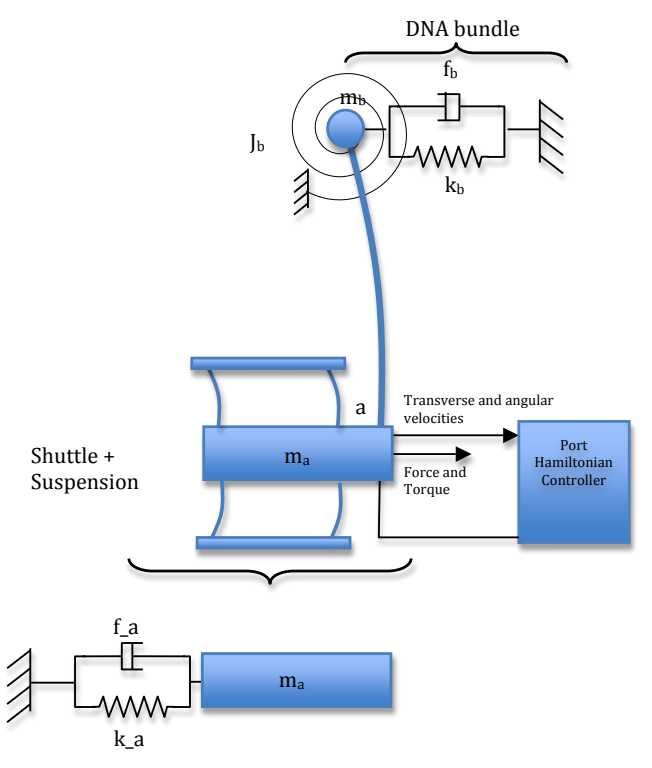

Fig. 2. DNA manipulation with $\mathrm{PH}$ control

The trapped DNA bundle is approximated by an equivalent mass spring damper system. We consider the arm of the tweezer is clamped in $z=a$. We also assume that it is only possible to measure the position at point $z=a$.

\subsection{Model of the tweezer arm}

The model of the tweezer arm is based on Timoshenko beam model. The Timoshenko beam has been widely studied as a distributed parameter port Hamiltonian system (Macchelli and Melchiorri, 2004) and as BCS (Le Gorrec et al., 2005). The model of the Timoshenko beam is written as:

$$
\frac{\partial}{\partial t}\left[\begin{array}{l}
x_{1} \\
x_{2} \\
x_{3} \\
x_{4}
\end{array}\right]=\underbrace{\left[\begin{array}{llll}
0 & 1 & 0 & 0 \\
1 & 0 & 0 & 0 \\
0 & 0 & 0 & 1 \\
0 & 0 & 1 & 0
\end{array}\right]}_{P_{1}} \frac{\partial}{\partial z}\left[\begin{array}{c}
K x_{1} \\
\frac{1}{\rho} x_{2} \\
E I x_{3} \\
\frac{1}{I_{\rho}} x_{4}
\end{array}\right]+\underbrace{\left[\begin{array}{cccc}
0 & 0 & 0 & -1 \\
0 & 0 & 0 & 0 \\
0 & 0 & 0 & 0 \\
1 & 0 & 0 & 0
\end{array}\right]}_{P_{0}}\left[\begin{array}{c}
K x_{1} \\
\frac{1}{-} x_{2} \\
\frac{E}{E I x_{3}} \\
\frac{1}{I_{\rho}} x_{4}
\end{array}\right]
$$

where the following state (energy) variables have been defined: $x_{1}=\frac{\partial w}{\partial z}(z, t)-\phi(z, t)$ the shear displacement, $x_{2}=\rho(z) \frac{\partial w}{\partial t}(z, t)$ the transverse momentum distribution, $x_{3}=\frac{\partial \phi}{\partial z}(z, t)$ the angular displacement, and $x_{4}=$ $I_{\rho} \frac{\partial \phi}{\partial t}(z, t)$ the angular momentum distribution, for $z \in$ $(a, b), t \geq 0$, where $w(t, z)$ is the transverse displacement 
of the beam and $\phi(t, z)$ is the rotation angle of a filament of the beam. The coefficients $\rho(z), I_{\rho}(z), E(z), I(z)$ and $K(z)$ are the mass per unit length, the rotary moment of inertia of a cross section, Young's modulus of elasticity, the moment of inertia of a cross section, and the shear modulus respectively. The matrices $P_{1}$ and $P_{0}$ defines the skewsymmetric differential operator of order 1 acting on the state space $X=L_{2}\left(a, b, \mathbb{R}^{4}\right), \mathcal{J}=P_{1} \frac{\partial}{\partial z}+P_{0}$. The energy of the beam is expressed in terms of the energy variables, $E=\frac{1}{2} \int_{a}^{b}\left(K x_{1}^{2}+\frac{1}{\rho} x_{2}^{2}+E I x_{3}^{2}+\frac{1}{I_{\rho}} x_{4}^{2}\right) d z=\frac{1}{2}\|x\|_{\mathcal{L}}^{2}$. The boundary port variables are obtained by using integration by parts and factorization in order to define an extended Dirac structure including the boundary (Le Gorrec et al., 2005 ). They also can be directly parametrized from $P_{1}$ (Le Gorrec et al., 2005; Villegas, 2007) leading to:

$$
\left[\begin{array}{c}
f_{\partial, \mathcal{L} x} \\
e_{\partial, \mathcal{L} x}
\end{array}\right]=\left[\begin{array}{c}
\left(\rho^{-1} x_{2}\right)(b)-\left(\rho^{-1} x_{2}\right)(a) \\
\left(K x_{1}\right)(b)-\left(K x_{1}\right)(a) \\
\left(I_{\rho}^{-1} x_{4}\right)(b)-\left(I_{\rho}^{-1} x_{4}\right)(a) \\
\left(E I x_{3}\right)(b)-\left(E I x_{3}\right)(a) \\
\left(\rho^{-1} x_{2}\right)(b)+\left(\rho^{-1} x_{2}\right)(a) \\
\left(K x_{1}\right)(b)+\left(K x_{1}\right)(a) \\
\left(I_{\rho}^{-1} x_{4}\right)(b)+\left(I_{\rho}^{-1} x_{4}\right)(a) \\
\left.\rho I x_{3}\right)(b)+\left(E I x_{3}\right)(a)
\end{array}\right] .
$$

The control objective is to control the translational position of the DNA-bundle. The physical ports are given by the translational force acting at the base of the beam (input), and the translational velocity at the base of the beam (output). All physical ports are hence located on the point $a$ of the beam and directly associated with the dynamic of the suspension mechanism and/or base of the beam. In order to achieve that the input and output variables of the flexible arm coincide with the physical ones we define the following input and outputs for the beam:

$$
u=[v(b) \omega(b)-v(a)-\omega(a)], \quad y=\left[\begin{array}{llll}
\Gamma(b) & T(b) & F(a) & T(a)
\end{array}\right],
$$

which is achieved by defining $u=W\left[\begin{array}{c}f_{\partial, \mathcal{L} x} \\ e_{\partial, \mathcal{L} x}\end{array}\right], y=$ $\tilde{W}\left[\begin{array}{l}f_{\partial, \mathcal{L} x} \\ e_{\partial, \mathcal{L} x}\end{array}\right]$ where

$$
W=\left[\begin{array}{cccccccc}
1 & 0 & 0 & 0 & 0 & 1 & 0 & 0 \\
0 & 0 & 1 & 0 & 0 & 0 & 0 & 1 \\
1 & 0 & 0 & 0 & 0 & -1 & 0 & 0 \\
0 & 0 & 1 & 0 & 0 & 0 & 0 & -1
\end{array}\right], \tilde{W}=\left[\begin{array}{cccccccc}
0 & 1 & 0 & 0 & 1 & 0 & 0 & 0 \\
0 & 0 & 0 & 1 & 0 & 0 & 1 & 0 \\
0 & -1 & 0 & 0 & 1 & 0 & 0 & 0 \\
0 & 0 & 0 & -1 & 0 & 0 & 1 & 0
\end{array}\right] .
$$

It can by shown that with this choice of input and output the system (20) defines an abstract boundary control system. Furthermore $\mathcal{A} x=P_{1}(\partial / \partial z)(\mathcal{L} x)+P_{0} \mathcal{L} x$ with domain $D(\mathcal{A})=\left\{\mathcal{L} x \in H^{1}\left(a, b ; \mathbb{R}^{n}\right) \mid\left[\begin{array}{c}f_{\partial, \mathcal{L} x} \\ e_{\partial, \mathcal{L} x}\end{array}\right] \in \operatorname{ker} W\right\}$ generates a contraction semigroup on $X$ and the energy balance equation is defined as: $\frac{d E}{d t}=u^{T} y$

\subsection{Finite dimensional controller model}

At point $b$ the DNA-bundle is represented by the simple Mass-spring-damper system of Figure 1 and thus admits a port Hamiltonian system representation. Then we can write by using $v_{b}=\left(x_{b}, m_{b} \dot{x}_{b}, \theta_{b}, m_{b} \omega_{b}\right)^{T}, u_{b}=$ $[F(b) T(b)]^{T}$ and $y_{b}=[v(b) \omega(b)]^{T}$ :

$$
\dot{v}_{b}=\left(J_{b}-R_{b}\right) \frac{d E_{b}}{d v_{b}}+g_{b} u_{b}, \quad y_{b}=g_{b}^{T} \frac{d E_{b}}{d v_{b}}
$$

with $E_{b}$ the energy of the system (sum of the kinetic and potential energies): $E_{b}\left(x_{b}, m_{b} \dot{x}_{b}, \theta_{b}, J_{b} \omega_{b}\right)=$ $\frac{k_{b}}{2} x_{b}^{2}+\frac{1}{2 M}\left(M \dot{x}_{c 2}\right)^{2}+\frac{k_{\theta_{b}}}{2} \theta_{b}^{2}+\frac{1}{2 J_{b}}\left(J_{b} \omega_{b}\right)^{2}$ and $J_{b}=$ $\left[\begin{array}{cccc}0 & 1 & 0 & 0 \\ -1 & 0 & 0 & 0 \\ 0 & 0 & 0 & 1 \\ 0 & 0 & -1 & 0\end{array}\right], R_{b}=\left[\begin{array}{cccc}0 & 0 & 0 & 0 \\ 0 & f_{b} & 0 & 0 \\ 0 & 0 & 0 & 0 \\ 0 & 0 & 0 & f_{\theta_{b}}\end{array}\right], g_{b}^{T}=\left[\begin{array}{cccc}0 & 1 & 0 & 0 \\ 0 & 0 & 0 & 1\end{array}\right]$, where $f_{b}$ and $f_{\theta_{b}}$ are the damping and the rotational damping constants at the interconnection point. At point a the shuttle is represented by a Mass-spring-damper system and is interconnected to the Port Hamiltonian Controller that basically acts as a programmable damping and stiffness. The resulting dynamic system is given by:

$$
\dot{v}_{a}=\left(J_{a}-R_{a}\right) \frac{d E_{a}}{d v_{a}}+g_{a} u_{a}, \quad y_{a}=g_{a}^{T} \frac{d E_{a}}{d v_{a}}
$$

with $E_{a}\left(x_{a}, m_{a} \dot{x}_{a}\right)=\frac{1}{2}\left(-k+k_{c}\right) x_{a}^{2}+\frac{1}{2 m_{b}}\left(m_{a} \dot{x}_{a}\right)^{2}$ the energy of the system. $J_{a}=\left[\begin{array}{cc}0 & 1 \\ -1 & 0\end{array}\right], R_{b}=\left[\begin{array}{cc}0 & 0 \\ 0 & f_{a}+f_{c}\end{array}\right], g_{a}^{T}=$ $\left[\begin{array}{lll}0 & 1 & 0 \\ 0 & 0 & 0\end{array}\right]$. Finally the overall finite dimensional system can be written:

$$
\dot{v}=\left(J_{c}-R_{c}\right) \frac{d E_{c}}{d v}+g_{c} u_{c}, \quad y_{c}=g_{c}^{T} \frac{d E_{c}}{d v}
$$

with $v=\left[x_{b}, m_{b} \dot{x}_{b}, \theta_{b}, J_{b} \omega_{b}, x_{a}, m_{a} \dot{x}_{a}\right], E_{c}(v)=$ $E_{b}\left(v_{b}\right)+E_{a}\left(v_{a}\right)$ and:

$$
J_{c}-R_{c}=\left[\begin{array}{cc}
J_{a}-R_{a} & 0 \\
0 & J_{b}-R_{b}
\end{array}\right], g_{c}=\left[\begin{array}{ll}
g_{a} & g_{b}
\end{array}\right]
$$

\subsection{Casimirs}

The Casimir functions are looked under the form (14) such that it satisfies equations (15), (16) and (17). More precisely the Casimir functions are constant functions (that do not depend on $t$ neither on $z$ ):

$$
C(x, v)=\kappa=\Gamma^{T} v+\int_{a}^{b} \Psi(z, t)^{T} x(z, t) d z
$$

satisfy:

- from condition (15):

$$
\begin{array}{r}
\Psi_{1}=C_{1} \\
\Psi_{2}=C_{4} z+C_{2} \\
\Psi_{3}=-C_{1} z+C_{3} \\
\Psi_{4}=C_{4}
\end{array}
$$

where $C_{i}, i \in[1, \cdots, 4]$ are constants.

- from condition (16):

$$
\begin{array}{r}
\Gamma_{2}=\Gamma_{4}=\Gamma_{6}=0 \\
\Gamma_{1}=-\Psi_{1}(b) \\
\Gamma_{3}=\Psi_{3}(b) \\
\Gamma_{5}=-\Psi_{1}(a)
\end{array}
$$

- from condition (17):

$$
\begin{array}{r}
\Gamma_{2}=-\Psi_{2}(b) \\
\Gamma_{4}=-\Psi_{4}(b) \\
\Gamma_{6}=-\Psi_{2}(a) \\
\Psi_{3}(a)=0
\end{array}
$$

From (21), (25) and (27), $C_{2}=C_{4}=0$ and $\Psi_{2}=\Psi_{4}=0$. Then (26) is satisfied. From (28) $C_{3}=-a C_{1}$ and then $\Gamma_{1}=-C_{1}, \Gamma_{3}=-C_{1}(a+b), \Gamma_{5}=C_{1}$ from (22), (23), and (24) respectively. Then the Casimir functions are defined as:

$\kappa=-C_{1} x_{b}-C_{1}(a+b) \Theta_{b}+C_{1} x_{a}+\int_{a}^{b} C_{1}\left(x_{1}-(z+a) x_{3}\right) d z$ 


\subsection{Control design}

The goal of the control law is to shape the total energy $E_{d}\left(v_{b}, x, v_{a}\right)$ such that it presents a minimum in the desired position of the tip of the arm, i.e.: $x_{b}^{*}=x_{b, c}^{*}$ and $\dot{x}_{b}^{*}=0,{\dot{\theta_{b}}}^{*}=0, \phi_{a}^{*}=0, \dot{\phi}_{a}^{*}=0$. The degrees of freedom we use for control design are the programmable "stiffness" and "damping" $k_{c}$ and $f_{c}$. The total energy is given by

$$
E_{d}\left(v_{b}, x, v_{a}\right)=E_{b}\left(x_{b}\right)+E(x)+E_{a}\left(x_{a}, p_{a}\right)
$$

From (29) we have:

$$
E_{d}\left(x_{b}, x, p_{a}\right)=E_{b}\left(x_{b}\right)+E(x)+E_{a}\left(F\left(x_{b}, x\right), p_{a}\right)
$$

Taking into account that

$$
E_{a}=\frac{1}{2}\left(f+k_{c}\right) x_{a}+\frac{1}{2 m_{a}} p_{a}^{2}
$$

we can write:

$$
\begin{array}{r}
\frac{\partial E_{d}}{\partial x}=\frac{\partial E}{\partial x}+\frac{\partial F^{T}}{\partial x} \frac{\partial E_{a}}{\partial x_{a}}=\frac{\partial E}{\partial x}+\Psi \frac{1}{2}\left(k_{a}+k_{c}\right) x_{a} \\
\frac{\partial E_{d}}{\partial v_{b}}=\frac{\partial E_{b}}{\partial v_{b}}+\Gamma \frac{1}{2}\left(k_{a}+k_{c}\right) x_{a} \\
\frac{\partial E_{d}}{\partial p_{a}}=\dot{x}_{a}
\end{array}
$$

$E$ admits a minimum in $\left(x_{b}^{*}, x^{*}, p_{a}^{*}\right)$ if equations $(30,31,32)$ equal zero for $\left(x_{b}, x, p_{a}\right)=\left(x_{b}^{*}, x^{*}, p_{a}^{*}\right)$. It is the case for (32) if $\dot{x}_{a}^{*}=0$. Using the notation $\alpha^{*}=\left(k_{a}+k_{c}\right) x_{a}^{*}$ we derive from (31) at the equilibrium:

and then

$$
k_{b} x_{b}^{*}+\Gamma_{1} \alpha^{*}=0, k_{\theta_{b}} \theta_{b}^{*}+\Gamma_{3} \alpha^{*}=0, \dot{x}_{b}^{*}=0
$$

$$
\theta_{b}^{*}=\frac{\Gamma_{3} k_{b}}{\Gamma_{1} k_{\theta_{b}}} x_{b}^{*}
$$

From (30) we deduce $x_{2}^{*}=x_{4}^{*}=0$ and:

$$
K x_{1}^{*}+\Psi_{1} \alpha^{*}=0, \quad E I x_{3}^{*}(z)+\Psi_{3}(z) \alpha^{*}=0
$$

Taking into account that $x_{3} *=\frac{\partial \phi^{*}(z)}{\partial z}$ we derive:

$$
\phi^{*}(z)=\int_{a}^{z}\left(-\frac{\alpha^{*}}{E I} \Psi_{3}\right) d z=\frac{C_{1} \alpha^{*}}{E I}\left(\frac{z^{2}}{2}+a z-\frac{3 a^{2}}{2}\right)
$$

and then

$$
\phi^{*}(b)=\frac{C_{1} \alpha^{*}}{2 E I}\left((a+b)^{2}-4 a^{2}\right)
$$

Furthermore $x_{1}^{*}=\frac{\partial \omega(z)}{\partial z}-\phi(z)$ and:

$$
w^{*}(z)=w^{*}(a)+x_{1}^{*}(z-a)+\frac{C_{1} \alpha^{*}}{2 E I}\left(\frac{z^{3}}{6}+\frac{a z^{2}}{2}-\frac{3 a^{2} z}{2}+\frac{5 a^{3}}{6}\right)
$$

Using the fact that the beam is clamped $\left(w^{*}(a)=0\right)$ to the moving shuttle we can write: $x_{a}^{*}=x_{b}^{*}+w^{*}(b)$ : and:

$$
x_{a}^{*}=\underbrace{x_{b}^{*}\left(1+\frac{k_{b}}{K}(b-a)+\frac{C_{1} k_{b}}{2 E I \Psi_{1}}\left(\frac{b^{3}}{6}+\frac{a b^{2}}{2}-\frac{3 a^{2} b}{2}+\frac{5 a^{3}}{6}\right)\right)}_{f\left(x_{b}^{*}\right)}
$$

and then:

$$
k_{c}\left(x_{b}^{*}\right)=-k_{a}-\frac{k_{b} x_{b}^{*}}{\Psi_{1} f\left(x_{b}^{*}\right)}
$$

Using (33) for $x_{b}^{*}=x_{b, c}^{*}$ allows to assign the desired closed loop equilibrium state. $f_{c}$ is designed in order to assign the dissipation rate. Indeed:

$$
\frac{d E_{d}}{d t}=-\frac{d E_{c}{ }^{T}}{d v} R_{c} \frac{d E_{c}}{d v}<-f_{c} x_{a}^{2}
$$

In this paper we considered a class of one dimensional boundary controller port Hamilonian systems interconnected in a energy preserving way to some port Hamiltonian controllers. During this interconnection we allowed clamping conditions by a rank deficiency of the input/output matrices of the controller. We proved the asymptotic stability of the closed loop system as soon as the controller is exponentially stable and derived some necessary conditions for the existence of structural invariants named Casimir functions. These Casimir can used to link the controller states to the system states in order to stabilize the system and shape the closed loop energy function to have a minimum at the desired state. This approach has been applied to a micromanipulation process leading to a simple Proportional Derivative control law. The perspective of this work is the generalization of such approach to non linear systems.

\section{REFERENCES}

Boudaoud, M., Haddab, Y., and Le Gorrec, Y. (2012). Modeling and optimal force control of a nonlinear electrostatic microgripper. Mechatronics, IEEE/ASME Transactions on, $\mathrm{PP}(99), 1-10$. doi: 10.1109/TMECH.2012.2197216.

Jacob, B. and Zwart, H. (2012). Linear Port-Hamiltonian Systems on Infinite-dimensional Spaces, volume 223 of Operator Theory: Advances and Applications. Birkhäuser, Basel, Switzerland.

John, F. (1978). Partial differential equations, volume 42.

Le Gorrec, Y., Zwart, H., and Maschke, B. (2005). Dirac structures and boundary control systems associated with skew-symmetric differential operators. SIAM Journal on Control and Optimization, 44(5), 18641892.

Macchelli, A. (2012). Boundary energy shaping of linear distributed port-Hamiltonian systems. In Proceedings of the 4th IFAC workshop on Lagrangian and Hamiltonian methods for non-linear control. Bertinoro, Italy.

Macchelli, A., Melchiorri, C., Pasumarthy, R., and van der Schaft, A.J. (2009). Modeling and Control of Complex Physical Systems - The Port-Hamiltonian Approach, chapter Analysis and Control of Infinite-Dimensional Systems, 319-368. Springer-Verlag, Berlin, Germany.

Macchelli, A. and Melchiorri, C. (2004). Modeling and control of the Timoshenko beam. the distributed port Hamiltonian approach. SIAM Journal on Control and Optimization, 43(2), 743-767.

Ortega, R., van der Schaft, A., Maschke, B., and Escobar, G. (2002). Interconnection and damping assignment passivity based control of port-controlled Hamiltonian systems. Automatica, 38, 585-596.

Ortega, R., van der Schaft, A.J., Mareels, I., and Maschke, B. (2001). Putting energy back in control. Control Systems Magazine, 21, 1833.

van der Schaft, A. (2004). Port-Hamiltonian systems: network modeling and control of nonlinear physical systems. In H. Irschik and K. Schlacher (eds.), Advanced Dynamics and Control of Structures and Machines, 127-168. Springer, New York.

van der Schaft, A.J. (2000). L2-Gain and Passivity Techniques in Nonlinear Control.

van der Schaft, A. and Maschke, B. (2002). Hamiltonian Formulation of Distributed Parameter Systems with Boundary Energy Flow. Journal of Geometry and Physics, 42, 166-194.

Villegas, J., Zwart, H., Le Gorrec, Y., and Maschke, B. (2009). Exponential stability of a class of boundary control systems. IEEE Transactions on Automatic Control, 54, 142-147.

Villegas, J., Zwart, H., Le Gorrec, Y., Maschke, B., and van der Schaft, A. (2005). Stability and stabilization of a class of boundary control systems. In Decision and Control, 2005 and 2005 European Control Conference. CDC-ECC' '05. 44th IEEE Conference on, 3850-3855.

Villegas, J.A. (2007). A port-Hamiltonian Approach to Distributed Parameter Systems. Ph.D. thesis, Universiteit Twente.

Willems, J.C. (1972). Dissipative dynamical systems Part II: Linear systems with quadratic supply rate, volume 45. 